\title{
Magnetically dominated structures as an important component of the solar wind turbulence
}

\author{
R. Bruno ${ }^{1}$, R. D'Amicis ${ }^{1}$, B. Bavassano ${ }^{1}$, V. Carbone ${ }^{2}$, and L. Sorriso-Valvo ${ }^{3}$ \\ ${ }^{1}$ INAF-Istituto Fisica Spazio Interplanetario, 00133 Roma, Italy \\ ${ }^{2}$ Dipartimento di Fisica Università della Calabria, 87036 Rende (Cs), Italy \\ ${ }^{3}$ LICRYL - INFM/CNR, 87036 Rende (Cs), Italy
}

Received: 21 February 2006 - Revised: 16 July 2007 - Accepted: 23 July 2007 - Published: 29 August 2007

\begin{abstract}
This study focuses on the role that magnetically dominated fluctuations have within the solar wind MHD turbulence. It is well known that, as the wind expands, magnetic energy starts to dominate over kinetic energy but we lack of a statistical study apt to estimate the relevance of these fluctuations depending on wind speed, radial distance from the sun and heliographic latitude. Our results suggest that this kind of fluctuations can be interpreted as non-propagating structures, advected by the wind during its expansion. In particular, observations performed in the ecliptic revealed a clear radial dependence of these magnetic structures within fast wind, but not within slow wind. At short heliocentric distances $(\sim 0.3 \mathrm{AU})$ the turbulent population is largely dominated by Alfvénic fluctuations characterized by high values of normalized cross-helicity and a remarkable level of energy equipartition. However, as the wind expands, a new-born population, characterized by lower values of Alfvénicity and a clear imbalance in favor of magnetic energy becomes visible and clearly distinguishable from the Alfvénic population largely characterized by an outward sense of propagation. We estimate that more than $20 \%$ of all the analyzed intervals of hourly scale within fast wind are characterized by normalized cross-helicity close to zero and magnetic energy largely dominating over kinetic energy. Most of these advected magnetic structures result to be non-compressive and might represent the crossing of the border between adjacent flux tubes forming, as suggested in literature, the advected background structure of the interplanetary magnetic field. On the other hand, their features are also well fitted by the Magnetic Field Directional Turnings paradigm as proposed in literature.
\end{abstract}

Keywords. Interplanetary physics (Plasma waves and turbulence; Solar wind plasma) - Space plasma physics (Turbulence)

Correspondence to: R. Bruno

(bruno@ifsi-roma.inaf.it)

\section{Introduction}

Belcher and Davis Jr. (1971) introduced, for the first time, the concept that propagating Alfvén modes could be intermixed with static structures advected by the solar wind. This idea came out from the observational evidence that Alfvénic correlations were less pure within slow velocity regions compared to high velocity streams. Afterwards, several authors corroborated this concept using additional experimental evidences (Bruno and Bavassano, 1991; Klein et al., 1993; Bruno and Bavassano, 1993) which showed that a high degree of Alfvénicity, intended as correlation between field $\delta \boldsymbol{b}$ and velocity $\delta \boldsymbol{v}$ fluctuations, is generally correlated with low compressibility of the medium although, in some cases, compressibility is not the only cause for a low Alfvénicity (Roberts et al., 1991, 1992; Roberts, 1992).

Detailed studies performed by Matthaeus et al. (1990) on the two-dimensional correlation function of solar wind fluctuations at $1 \mathrm{AU}$, generally known in literature as the "Maltese Cross", showed that interplanetary turbulence was made of two primary ingredients, namely Alfvénic fluctuations and 2-D turbulence. This two-dimensional turbulence is characterized for having both the wave vector $\boldsymbol{k}$ and the perturbing field $\delta \boldsymbol{b} \perp$ to the ambient field $\boldsymbol{B}_{\mathbf{0}}$. However, given that the analysis did not separate fast from slow wind, it is likely that most of the the slab (Alfvénic) correlations came from the fast wind while the 2-D correlations came from the slow wind. Anisotropic turbulence has been studied both theoretically (Montgomery, 1982; Zank and Matthaeus, 1992) and through numerical simulations (Shebalin et al., 1983; Oughton et al., 1994). In particular, these simulations focused on non-linear spectral transfer within MHD turbulence in presence of a strong mean magnetic field. It was observed that a remarkable anisotropy is created during the turbulent process and much of the power is transferred to fluctuations with higher $\boldsymbol{k}_{\perp}$ and less to fluctuations with higher $\boldsymbol{k}_{\|}$. Further theoretical models (Marsch and Tu, 1989; Tu and

Published by Copernicus Publications on behalf of the European Geosciences Union. 
Marsch, 1990; Zhou and Matthaeus, 1989) showed that the existence of 2-D non compressive turbulence comes out naturally from the structure of the transport equations. Bieber et al. (1996) formulated an observational test to distinguish the slab (Alfvénic) from the 2-D component within interplanetary turbulence. Several tests conducted by these authors assigned a fraction of $\sim 80 \%$ to the 2-D component and the remaining percentage to the Alfvénic or slab component. However, data used for this analysis were recorded near times of solar energetic particle events. Moreover, most of the data belonged to slow solar wind (Wanner and Wibberenz, 1993) and as such, this analysis is not representative of the whole phenomenon of turbulence in the solar wind. As a matter of fact, using Ulysses observations at high latitude, when the s/c was imbedded in the polar fast wind, Smith (2003) found that in these conditions the percentage of slab and 2-D components was about the same, say the high latitude slab component results unusually higher as compared to ecliptic observations Horbury et al. (2005).

The decrease of Alfvénicity has also been associated with a particular type of uncompressive events which $\mathrm{Tu}$ and Marsch (1991) discovered and called Magnetic Field Directional Turnings or MFDTs. A single case study, which might belong to the 2-D turbulence, was described during a study performed within slow wind (Tu and Marsch, 1991) and was characterized by very low value of normalized crosshelicity $\sigma_{C}$, close to zero, and low values of the Alfvén ratio $r_{A}$, around 0.2. We like to remind the reader that while $\sigma_{C}=\left(e^{+}-e^{-}\right) /\left(e^{+}+e^{-}\right)$measures the predominance of the energy associated to one of the two possible Alfvén modes $e^{+}$or $e^{-}, r_{A}$ measures the ratio between kinetic $E_{k}$ and magnetic energy $E_{b}$ of the fluctuations at a given time scale. This interval was only weakly compressive, and short period fluctuations, from a few minutes to about $40 \mathrm{~min}$, imbedded in it were nearly pressure balanced. In these structures most of the fluctuating energy resides in the magnetic field rather than velocity with the consequence that the energies associated to $e^{+}$and $e^{-}$result to be comparable and, consequently, the derived parameter $\sigma_{C} \rightarrow 0$. Tu and Marsch (1991) suggested that these fluctuations might derive from a special kind of magnetic structures, which obey the MHD equations, for which $(\boldsymbol{B} \cdot \nabla) \boldsymbol{B}=0$, magnetic field magnitude, wind velocity, proton density and temperature are all constant. However, as remarked by Tu and Marsch (1995), such a description is too simple since velocity fluctuations $\delta V$ are never 0 and Sigmac is never exactly -1 . Another way to call these static, advected structures suggested by these authors is "tangential turnings" which might be considered as the large scale counterpart of tangential discontinuities (TDs hereafter), just like Alfvén waves are the counterpart of rotational discontinuities.

The same authors suggested the possibility of an interplanetary turbulence mainly made of outwardly propagating Alfvén waves and advected structures represented by MFDTs (Tu and Marsch, 1993). In other words, this model assumed that the spectrum of $e^{-}$would be caused by MFDTs. The different radial evolution of the power associated with these two kind of components would determine the radial evolution observed in both $\sigma_{C}$ and $r_{A}$. However, the results of this model (Tu and Marsch, 1993) showed only a qualitative agreement with the observations and, we like to remark, we still do not have a convincing solution able to explain why $r_{A}$ tends to a value less than unit during the wind expansion (see ample discussion and references reported in Bruno and Carbone, 2005, and also recent results from direct numerical simulations (Müller and Grappin, 2005)).

Although Tu and Marsch (1991) concluded their paper with the recommendation to carry out more case studies and statistical studies on the existing data sets to establish the real importance of these structures in the solar wind turbulence, the first studies, to our knowledge, which statistically located a whole family of magnetically dominated structures, within the zoo of interplanetary MHD fluctuations, were presented by Bavassano et al. $(1998,2000)$. These authors studied the distributions of the events characterized by given values of normalized cross-helicity $\sigma_{C}$ and normalized residual energy $\sigma_{R}=\left(E_{k}-E_{b}\right) /\left(E_{k}+E_{b}\right)$ for different heliospheric regions crossed by Ulysses during its first orbit. They discovered that a predominance of outward fluctuations (positive values of $\sigma_{C}$ ) and of magnetic fluctuations (negative values of $\sigma_{R}$ ) represented a general feature of interplanetary MHD fluctuations. Besides the fact that the most Alfvénic region resulted to be the one at high latitude and at shorter heliocentric distance, in all the analyzed regions they clearly found a relative peak of the distribution at $\sigma_{C} \simeq 0$ and $\sigma_{R} \simeq-1$ strongly reminiscent of those magnetic structures found by Tu and Marsch (1991) in the ecliptic, namely the MFDTs. Bavassano et al. (1998) stressed the necessity to perform a systematic study in the inner heliosphere "to get a term of comparison with Ulysses results and hence improve our knowledge about their features and their dependence on wind speed regime and radial distance."

Following this recommendation, we performed a study similar to that of Bavassano et al. (1998) in the ecliptic, between 0.3 and $1 \mathrm{AU}$, and within the southernmost latitudinal excursion of Ulysses, not analyzed by these authors. Results relative to this analysis will be reported and discussed in the following sections.

\section{Data analysis}

In the following we will illustrate results obtained from the analysis of data recorded by Helios 2 in the inner heliosphere between $\sim 0.3$ and $\sim 0.9$ AU in 1976, WIND at the Lagrangian point [1 AU] during 1995/1996 and, finally, Ulysses during its south passage at high latitude in 1994. In particular, we like to stress that all the time periods considered in this analysis belong to minimum phases of the solar activity cycle. 
Table 1. From top to bottom, selected time intervals for Helios 2, WIND and Ulysses spacecraft, respectively. The analysed time interval, heliocentric distance and latitude, average solar wind speed, number of analysed data points and length of data average are shown from left to right. The specification "fast" and "slow" adopted for WIND indicates that the analysis has been performed for solar wind samples faster than $550 \mathrm{~km} / \mathrm{s}$ or slower than $400 \mathrm{~km} / \mathrm{s}$, respectively.

\begin{tabular}{|c|c|c|c|c|c|c|}
\hline $\mathrm{s} / \mathrm{c}$ & $\begin{array}{c}\text { time interval } \\
\text { [yy.ddd:hh-yy.ddd:hh] }\end{array}$ & $\begin{array}{l}\text { radial distance } \\
{[\mathrm{AU}]}\end{array}$ & $\begin{array}{c}\text { heliographic } \\
\text { latitude }\left[^{\circ}\right]\end{array}$ & $\begin{array}{l}<|\boldsymbol{V}|> \\
{[\mathrm{km} / \mathrm{s}]}\end{array}$ & No. samples & $\begin{array}{c}\text { Length of } \\
\text { data average [s] }\end{array}$ \\
\hline Helios2 & 76.46:00-76.48:00 & 0.90 & -6.36 & 433 & 2109 & 81 \\
\hline Helios2 & $76.49: 00-76.52: 12$ & 0.88 & -6.55 & 637 & 3685 & 81 \\
\hline Helios2 & $76.72: 00-76.74: 00$ & 0.69 & -7.23 & 412 & 2095 & 81 \\
\hline Helios 2 & $76.74: 18-76.77: 18$ & 0.65 & -7.23 & 627 & 3152 & 81 \\
\hline Helios 2 & 76.101:00-76.103:00 & 0.32 & -1.08 & 368 & 1751 & 81 \\
\hline Helios2 & $76.104: 12-76.110: 00$ & 0.29 & 3.16 & 716 & 5647 & 81 \\
\hline WIND-fast & 95.1:00-96.121:00 & 1.00 & $-7.25: 7.25$ & 621 & 26231 & 240 \\
\hline WIND-slow & $95.1: 00-96.121: 00$ & 1.00 & $-7.25: 7.25$ & 350 & 77220 & 240 \\
\hline Ulysses & 94.190:00-94.330:00 & $2.75: 1.77$ & $-72:-62$ & 745 & 24819 & 480 \\
\hline
\end{tabular}

\subsection{Helios 2 observations between 0.3 and $0.9 \mathrm{AU}$}

The present data analysis is based on $81 \mathrm{~s}$ averages of solar wind parameters recorded by Helios 2 during its primary mission to the sun in 1976 when the s/c was lucky enough to repeatedly observe the same corotating stream at three different heliocentric distances, during three consecutive solar rotations (Bavassano et al., 1982b). For each stream we selected a time interval within the trailing edge and a slow wind interval ahead of it, having care of avoiding to include the stream-stream interface within which dynamical interaction mainly develops. The selected intervals, the relative average heliocentric distance and the average solar wind speed are reported in Table 1. For each of these data sets, we built the three components of both Elsässer variables $z^{+}$and $z^{-}$. Following Elsässer (1950); Dobrowolny et al. (1980); Goldstein et al. (1986); Grappin et al. (1989); Marsch and Tu (1989); Tu and Marsch (1990); Tu et al. (1989), in a plasma populated by Alfvén modes, it is useful to introduce the Elsässer variables which are defined as

$z^{ \pm}=v \pm \frac{b}{\sqrt{4 \pi \rho}}$

where $\boldsymbol{v}$ and $\boldsymbol{b}$ are the proton velocity and the magnetic field measured in the $\mathrm{s} / \mathrm{c}$ reference frame which can be looked at as an inertial reference frame and $\rho$ the plasma density. The sign in front of $\boldsymbol{b}$, in Eq. (1), depends on $\operatorname{sign}\left[-\boldsymbol{k} \cdot \boldsymbol{B}_{0}\right]$. In other words, for an outward directed mean field $\boldsymbol{B}_{0}$, a negative correlation would indicate an outward directed wave vector $\boldsymbol{k}$ and vice-versa. However, it is more convenient to define the Elsässers variables in such a way that $z^{+}$always refers to waves moving outward and $z^{-}$to waves moving inward. In order to do so, the magnetic field of each single $81 \mathrm{~s}$ average was artificially rotated by $180^{\circ}$ every time its projection on the background magnetic field $\boldsymbol{B}_{0}$ pointed away from the sun along $\boldsymbol{B}_{0}$, in other words, magnetic sectors were rectified (Roberts et al., 1987a,b).
Successively, we followed a type of analysis similar to that of Bavassano et al. (1998). We computed the normalized cross helicity $\sigma_{C}$ and the residual energy $\sigma_{R}$ within a window of $1 \mathrm{~h}$ that was repeatedly shifted by $81 \mathrm{~s}$ across the whole data set. Although this procedure (not adopted by Bavassano et al., 1998), makes the $1 \mathrm{~h}$ sub-intervals not longer independent on each other, it considerably enhances the statistics by a factor larger than 44 , without altering the validity of the results that, as we checked, fully reflect the results that we would obtain using independent $1 \mathrm{~h}$ sub-intervals.

The first results we report in Fig. 1 show the 2-D histogram of $e^{-}$versus $e^{+}$in $\log$-Log scale, being $e^{-}$and $e^{+}$the power associated to $\delta z^{-}$and $\delta z^{+}$fluctuations, computed from the trace of the corresponding variance matrix of the components. These variances were discarded whenever the number of elements within each $1 \mathrm{~h}$ interval was less than $30 \%$ of the total number, i.e. less than 14 . The three panels of this figure refer, from top to bottom, to results obtained for the fast wind observed at $0.29,0.65$ and $0.88 \mathrm{AU}$ (Table 1), respectively. Moreover, the colored straight lines indicate constant values of $\sigma_{C}$. The distribution, which at $0.29 \mathrm{AU}$ shows a well defined single peak between 0.9 and $0.95 \sigma_{C}$ lines, becomes less defined at $0.65 \mathrm{AU}$, shifts the main core of the distribution towards smaller values of $e^{+}$and starts to develop a tail towards much lower values of $\sigma_{C}$. This tendency becomes a clear evidence at $0.88 \mathrm{AU}$ when a second distribution is established along the $\sigma_{C} \sim 0$ line. This second family is located above the main distribution and is due to enhanced values of $e^{-}$rather than to depleted values of $e^{+}$. If we try to estimate the number of values forming the upper distribution, choosing $\sigma_{C}=0.5$ as the border line between the two distributions, we come out with a remarkable fraction of $\sim 30 \%$ of the total number of elements.

Because of its definition, $\sigma_{C}=0 \Longrightarrow e^{+}=e^{-}$and, in turn, $e^{+}=e^{-} \Longrightarrow \delta \boldsymbol{v} \perp \delta \boldsymbol{b}$, i.e. complete misalignment, or $E_{b}$ or $E_{k}=0$. In order to understand the role played by magnetic 

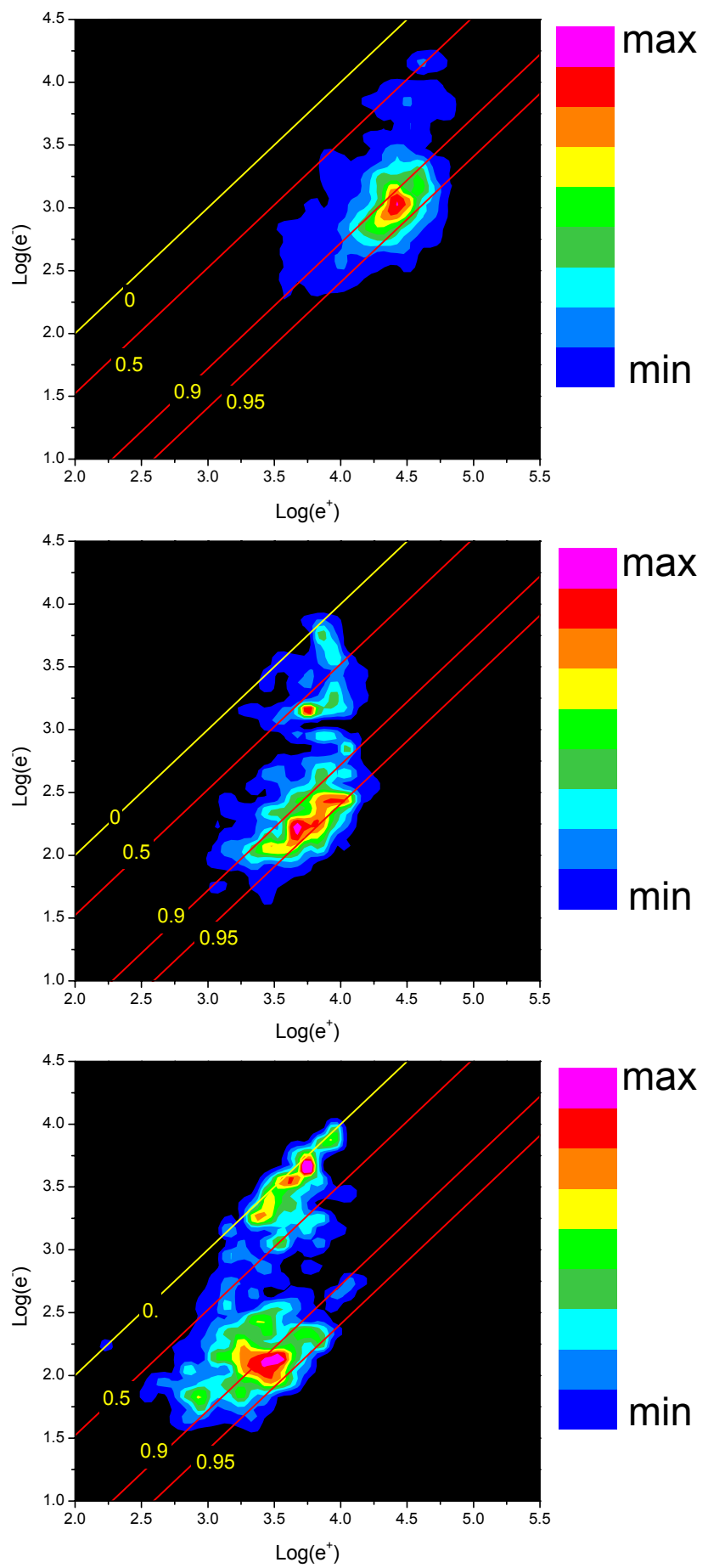

Fig. 1. From top to bottom: frequency histograms of $\log \left(e^{-}\right)$versus $\log \left(e^{+}\right)$for fast wind observed by Helios 2 at $0.29,0.65$ and $0.88 \mathrm{AU}$, respectively. The color code, for each panel, is normalized to the maximum of the distribution. Colored straight lines indicate constant values of $\sigma_{C}$.

and kinetic energy in the previous distributions, we built a 2-D histogram of $\sigma_{R}$ versus $\sigma_{C}$ values for the same data in-
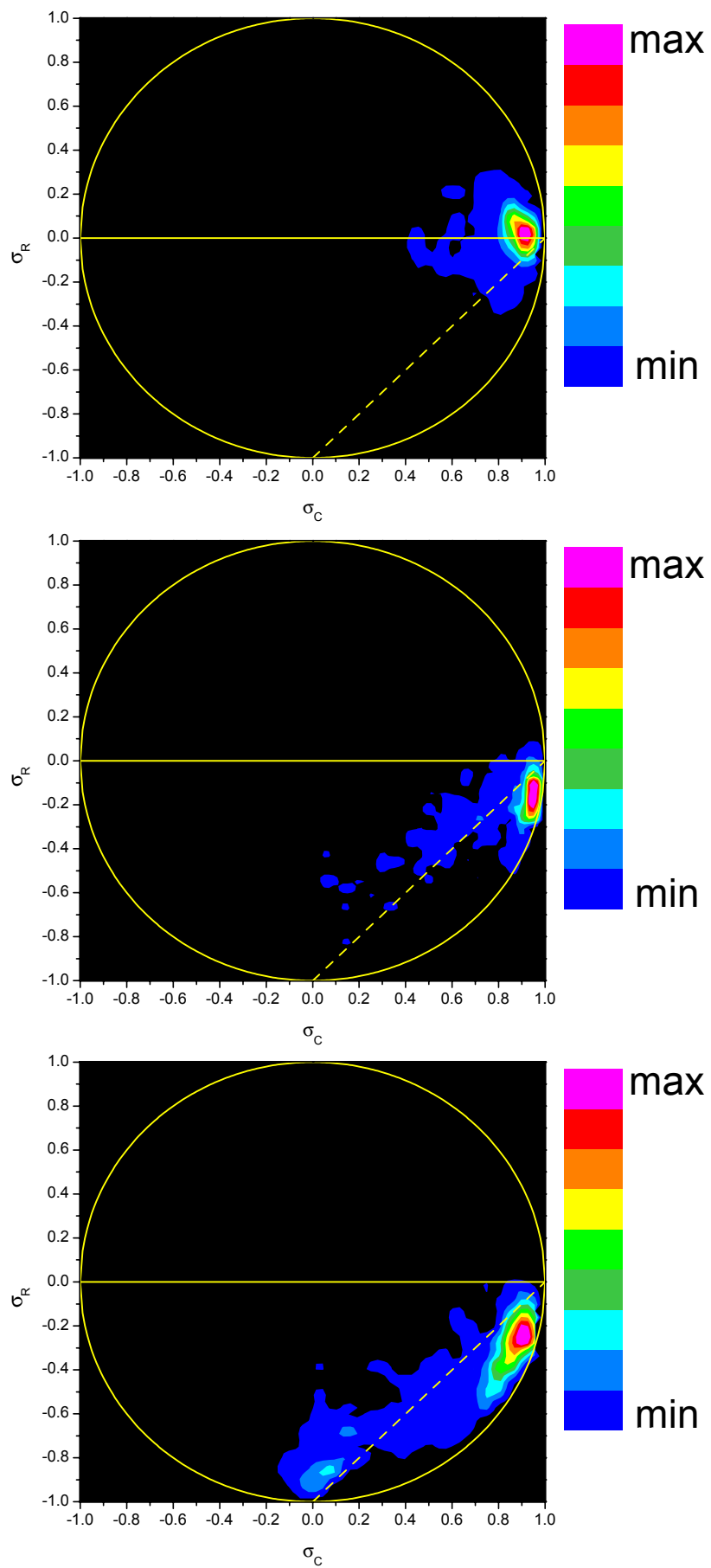

Fig. 2. From top to bottom: frequency histograms of $\sigma_{R}$ versus $\sigma_{C}$ for fast wind observed by Helios 2 at $0.29,0.65$ and $0.88 \mathrm{AU}$, respectively. The color code, for each panel, is normalized to the maximum of the distribution. The yellow circle represents the limiting value given by $\sigma_{C}^{2}+\sigma_{R}^{2}=1$ while, the yellow dashed line represents the relation $\sigma_{R}=\sigma_{C}-1$, see text for details. .

tervals analyzed in Fig. 1. These results, shown in Fig. 2, highlight the presence of a radial evolution of the fluctuations 
towards a double-peaked distribution during the expansion of the wind. While at $0.3 \mathrm{AU}$ the peak of the distribution, well centered around $\sigma_{R} \sim 0$ and $\sigma_{C} \sim 1$, suggests the overwhelming presence of outwardly propagating Alfvén modes, the distribution corresponding to $0.7 \mathrm{AU}$ denotes the formation of a tail towards negative values of $\sigma_{R}$ and lower values of $\sigma_{C}$ with a consequent depletion of the main peak with respect to the whole distribution. Moreover, this peak loses some of the Alfvénic character it showed in the previous panel since it is located at negative $\sigma_{R}$. This tendency ends up with the appearance of a sort of secondary peak when the wind reaches $0.88 \mathrm{AU}$. This new born peak forms around $\sigma_{R} \sim-1$ and $\sigma_{C} \sim 0$. These characteristics suggest that this new-born family might be made of MFDT structures just like those found by Tu and Marsch (1991). Parallel to the appearance of these fluctuations, the main peak characterized by Alfvénlike fluctuations moves to lower and lower values of $\sigma_{R}$ and $\sigma_{C}$ loosing much of the original character it had at $0.3 \mathrm{AU}$. At this point, we can introduce a new limit on $\sigma_{R}$ in order to better identify those fluctuations which do not have Alfvénic character and are characterized by a clear excess of magnetic energy. Thus, if we count all the intervals for which $\sigma_{C}<0.5$ and $\sigma_{R}<-0.6$ we end up with a population of about $23 \%$ of the total number of intervals.

In the next Fig. 3 we take a look at one of these events located within the trailing edge of the high velocity stream observed at $0.88 \mathrm{AU}$. The three panels, from top to bottom, show the $81 \mathrm{~s}$ fluctuations of the three components of velocity (black solid line) and magnetic field (red solid line), expressed in Alfvén units, relative to their respective average values. The two vertical dashed lines highlight a subinterval lasting more than $1 \mathrm{~h}$ during which magnetic fluctuations largely dominate over kinetic fluctuations. Moreover, it is interesting to notice how this interval is located between two regions characterized by much better Alfvénic correlations and energy equipartition. As a matter of fact, this central region shows also a higher level of variability in terms of magnetic field intensity, plasma density and temperature (not shown here) which would suggest a sort of pressure balance structure. Together with these relatively short intervals there are also much longer time intervals during which magnetic energy dominates and Alfvénic correlation is very low. One of these intervals is shown in Fig. 4 and is taken from the trailing edge of the same high velocity stream previously discussed. This case differs from the one shown before mainly because it lasts several hours and closely resembles the events shown by Tu and Marsch (1991) that they called MFDTs.

Tu and Marsch (1991) made the hypothesis that fluctuations were made solely by Alfvén waves outwardly propagating and advected MFDTs, and derived a simple expression relating the Alfvén ratio $r_{A}=E_{k} / E_{b}$ to $\sigma_{C}$

$r_{A}=\sigma_{C} /\left(2-\sigma_{C}\right)$

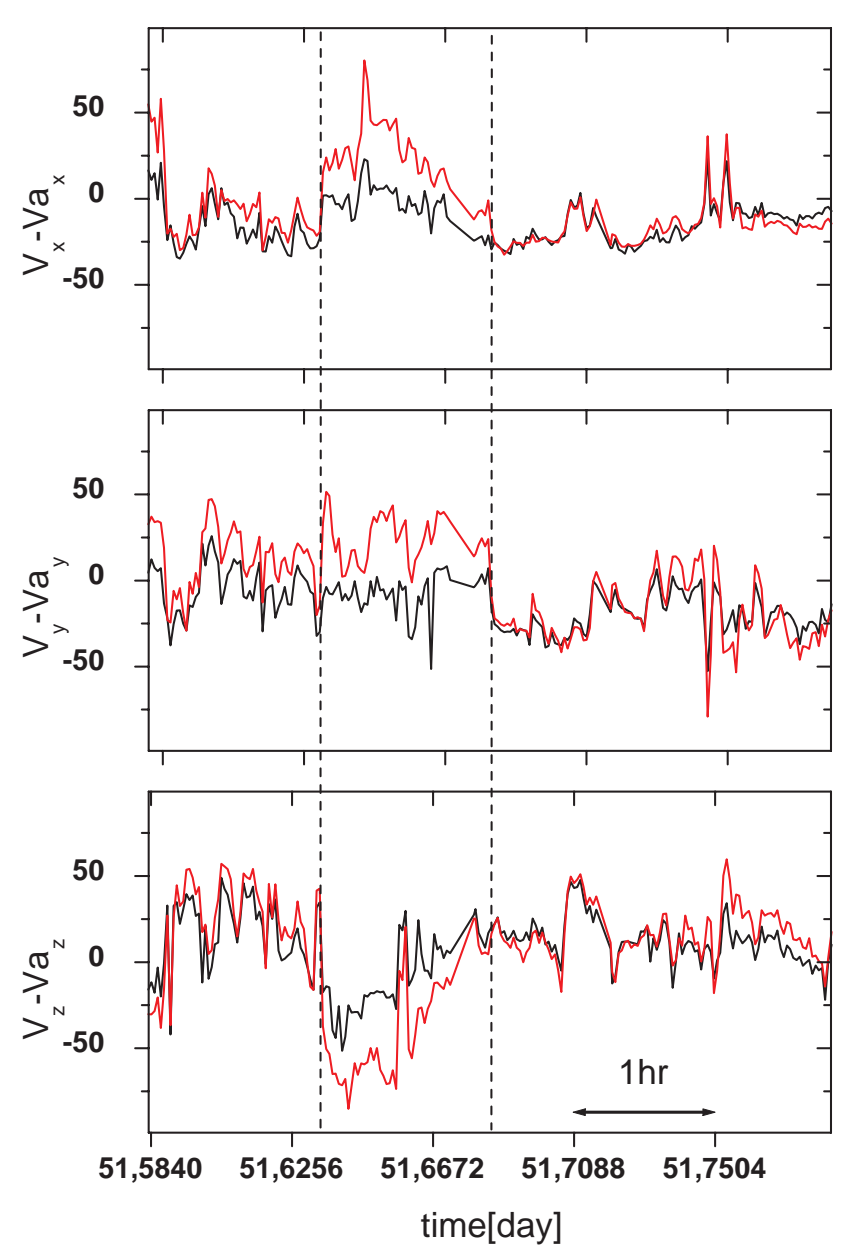

Fig. 3. From top to bottom: $81 \mathrm{~s}$ fluctuations of the three components of velocity (black solid line) and magnetic field (red solid line), expressed in Alfvén units, relative to their respective average values. This time interval belongs to the trailing edge of a high speed stream observed at $0.88 \mathrm{AU}$. Distance between two consecutive tick marks on the "X" axis corresponds to $1 \mathrm{~h}$.

which, however, only qualitatively agreed with Helios observations, as can be seen from their Fig. 8. This expression, taking into account that the normalized residual energy $\sigma_{R}$ can be expressed in terms of $r_{A}$ as $\sigma_{R}=\left(r_{A}-1\right) /\left(r_{A}+1\right)$, would also bring the following linear relation between $\sigma_{R}$ and $\sigma_{C}$ (Bavassano et al., 1998):

$\sigma_{R}=\sigma_{C}-1$

which would replace the canonical quadratic relation $\sigma_{R}^{2}+\sigma_{C}^{2} \leq 1$ and would be satisfied by a magnetic field vector $\boldsymbol{b}=\boldsymbol{v}+\boldsymbol{c}$ where $\boldsymbol{v}$ is the velocity vector and $\boldsymbol{c}$ an arbitrary vector $\perp \boldsymbol{v}$ and due to MFDTs. These magnetic fluctuations would then alter the $\boldsymbol{b}, \boldsymbol{v}$ alignment due to the presence of outwardly propagating Alfvénic fluctuations, reducing the value of $\sigma_{C}$. However, the yellow dashed line shown in the three panels of Fig. 2, which refers to Eq. (2), does not seem 

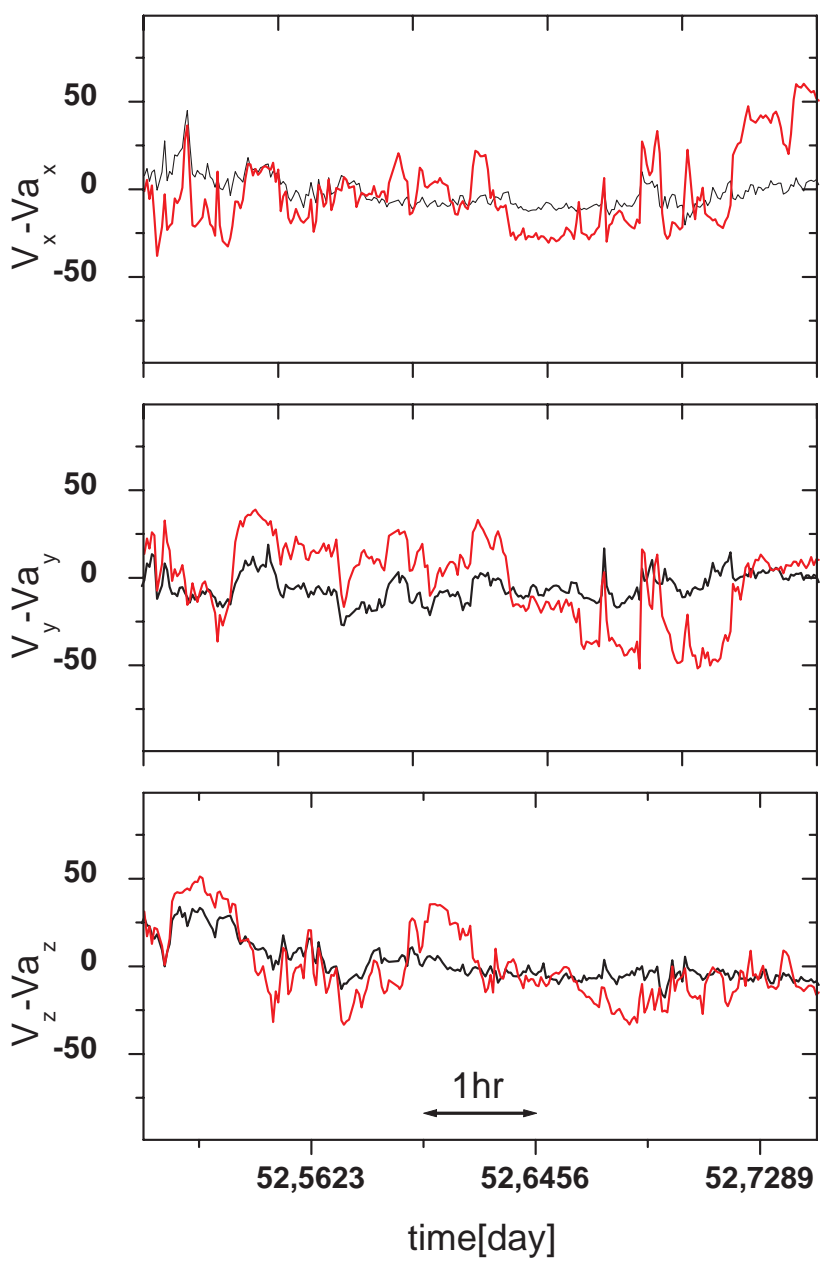

Fig. 4. From top to bottom: $81 \mathrm{~s}$ fluctuations of the three components of velocity and magnetic field in the same format as of Fig. 3. Also this time interval belongs to the trailing edge of the same high speed stream.

to provide a satisfactory, quantitative fit to the distribution. This result confirms similar conclusions drawn by $\mathrm{Tu}$ and Marsch (1991) in their original analysis.

Another way to look at the same data is shown in Fig. 5 where the average value of $\sigma_{R}$ computed for each square bin $\log \left(e^{-}\right) \times \log \left(e^{+}\right)$is shown for the same three time intervals. The color code indicates that only fluctuations observed at the shortest heliocentric distance (top panel) are characterized by values of $\sigma_{R}$ close to equipartition between $E_{b}$ and $E_{k}$. As the wind expands, $\sigma_{R}$ becomes more and more negative although high values of $\sigma_{C}$ around the 0.95 line are still present even at $0.88 \mathrm{AU}$ (bottom panel). Finally, the new population that clearly appears at $0.88 \mathrm{AU}$ around $\sigma_{C} \sim 0$, is totally characterized by values of $\sigma_{R} \sim-1$, indicating that magnetic field fluctuations dominate the turbulent dynamics.

At this point, it is interesting to look at the compressive level of these fluctuations as shown in Fig. 6. The upper panel of Fig. 6 shows the histogram of magnetic field
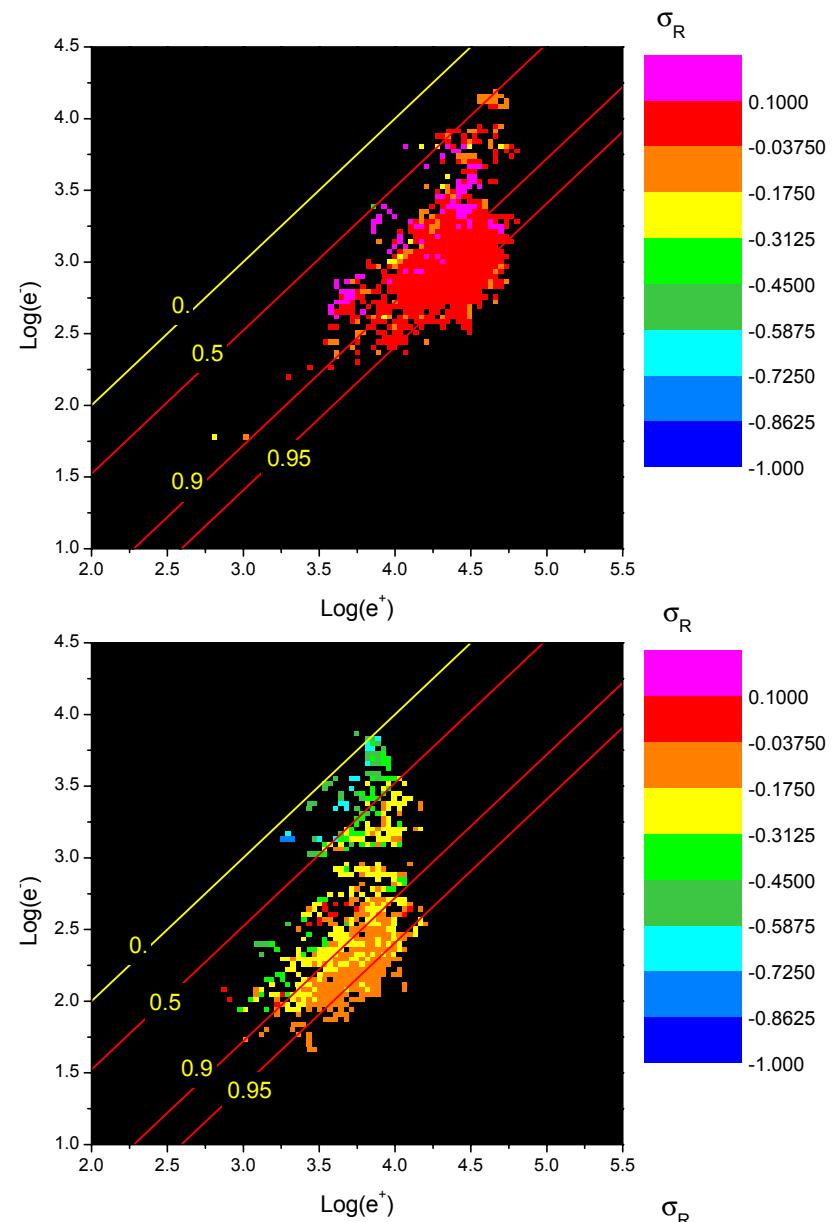

4500
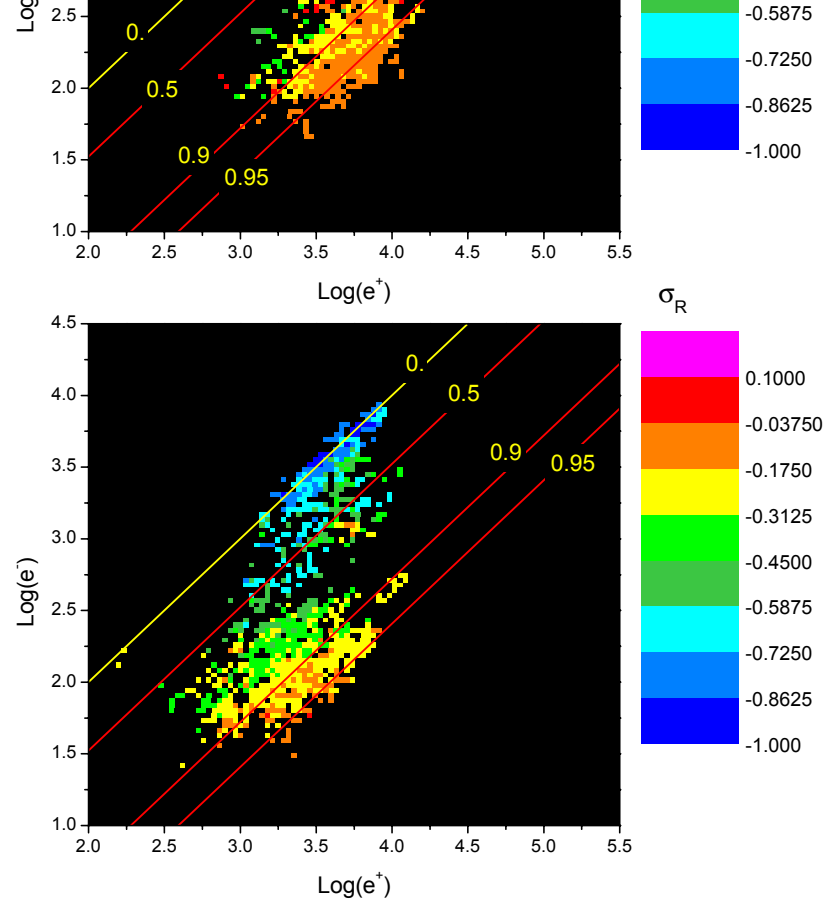

Fig. 5. From top to bottom: distribution of the average value of $\sigma_{R}$ computed within each square bin $\log \left(e^{-}\right) \times \log \left(e^{+}\right)$for fast wind observed by Helios 2 at $0.29,0.65$ and $0.88 \mathrm{AU}$, respectively. The color code, for each panel, indicates the interval value of $\sigma_{R}$.

compression defined as $S_{B}=\sqrt{\sigma_{B}^{2} /<|\boldsymbol{B}|>^{2}}$ as a function of $\sigma_{R}$ versus $\sigma_{C}$ while, the lower panel shows the histogram of $F_{C}=\sigma_{B}^{2} / \sigma_{x y z}^{2}$ which measures the ratio of the variance of the magnitude to the total variance of the vector, being $\sigma_{B}$ 


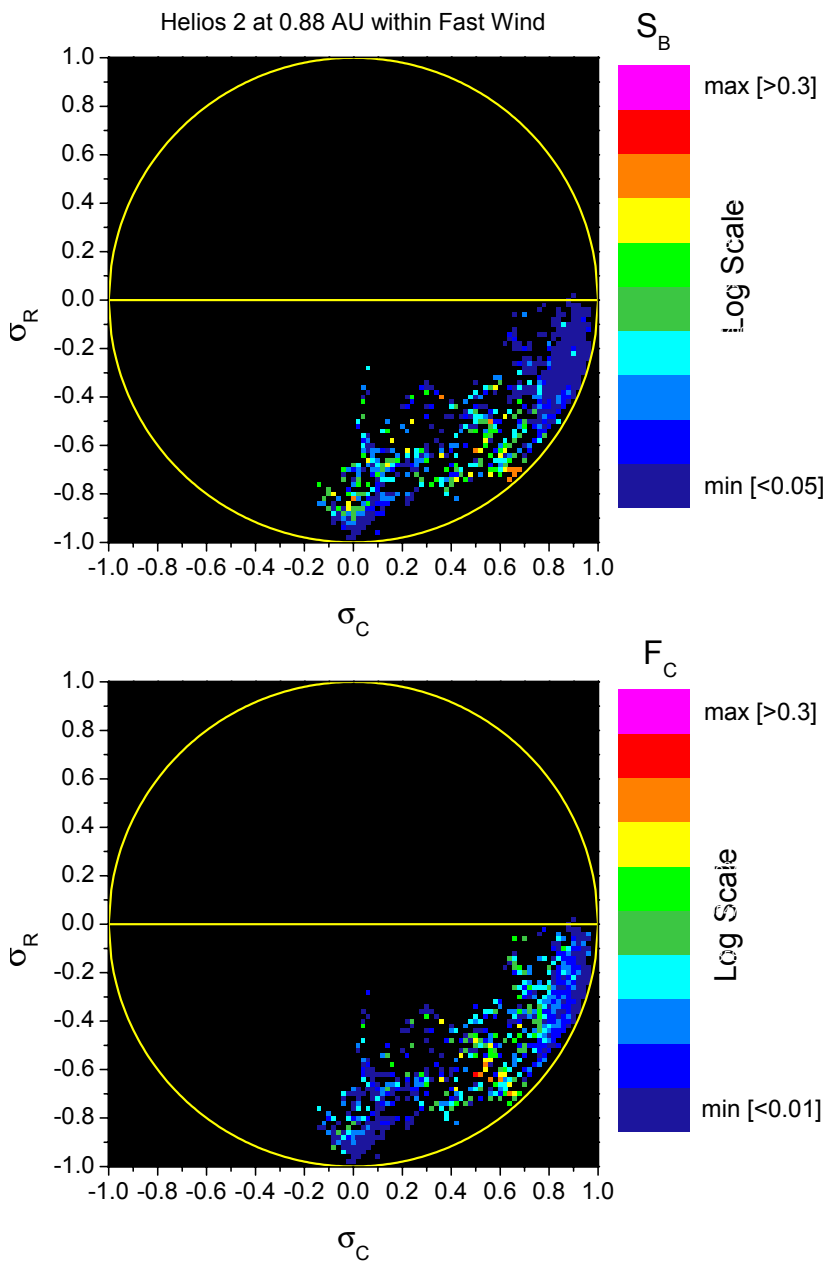

Fig. 6. From top to bottom: average value of $S_{B}$ and $F_{C}$ (see text for definitions) computed within each square bin $\sigma_{R} \times \sigma_{C}$ for Helios 2 observations at $0.88 \mathrm{AU}$ within fast wind.

and $\sigma_{x y z}^{2}$ the standard deviation of the magnetic field intensity and the trace of the variance matrix of the components, respectively (Bavassano et al., 1982a; Bruno and Bavassano, 1991). Both panels clearly indicate that the two regions characterized by $\sigma_{C} \sim 1$ and $\sigma_{R} \sim 0$ and $\sigma_{C} \sim 0$ and $\sigma_{R} \sim-1$, respectively, show the lowest magnetic compressive level. In particular, the lower panel indicates that most of the total vector variability is due to directional rather than compressive fluctuations, being this aspect, as already remarked in this paper, an intrinsic feature of Alfvénic fluctuations and MFDTs. Thus these results would support the idea that these two populations would be due mainly to Alfvénic outward fluctuations and MFDTs (Tu and Marsch, 1991, 1993), respectively. However, we cannot exclude that also Tangential Discontinuities, whose rate is expected to be at least one per hour (Tsurutani and Smith, 1979), might contribute to enhance the imbalance in favor of magnetic energy.
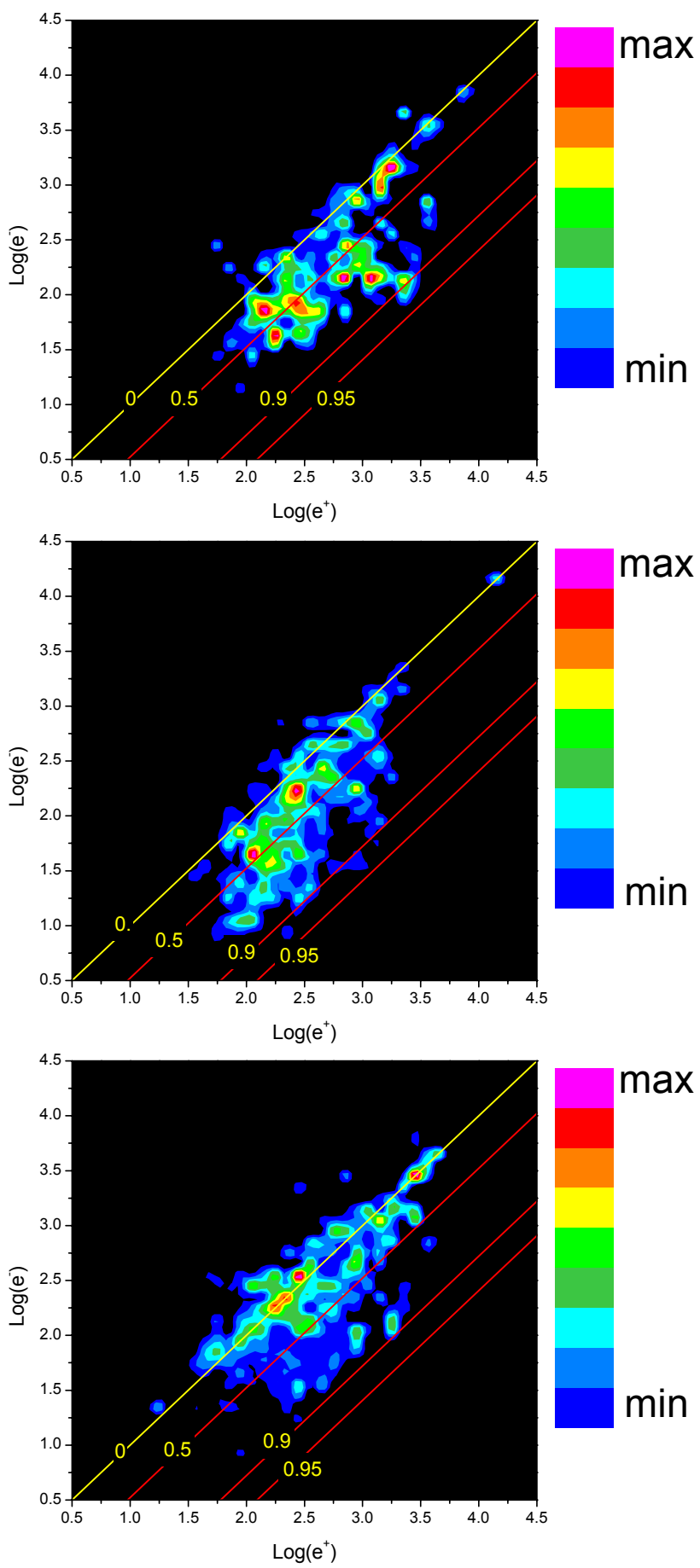

Fig. 7. From top to bottom: frequency histograms of $\log \left(e^{-}\right)$versus $\log \left(e^{+}\right)$for slow wind observed by Helios 2 at $0.32,0.69$ and $0.90 \mathrm{AU}$, respectively. The color code, for each panel, is normalized to the maximum of the distribution.

Completely different is the situation within slow wind as shown in Fig. 7 where the 2-D histograms of $\log \left(e^{-}\right)$versus $\log \left(e^{+}\right)$refer to the three intervals listed in Table 1. First of all, the distributions are quite spread and already 

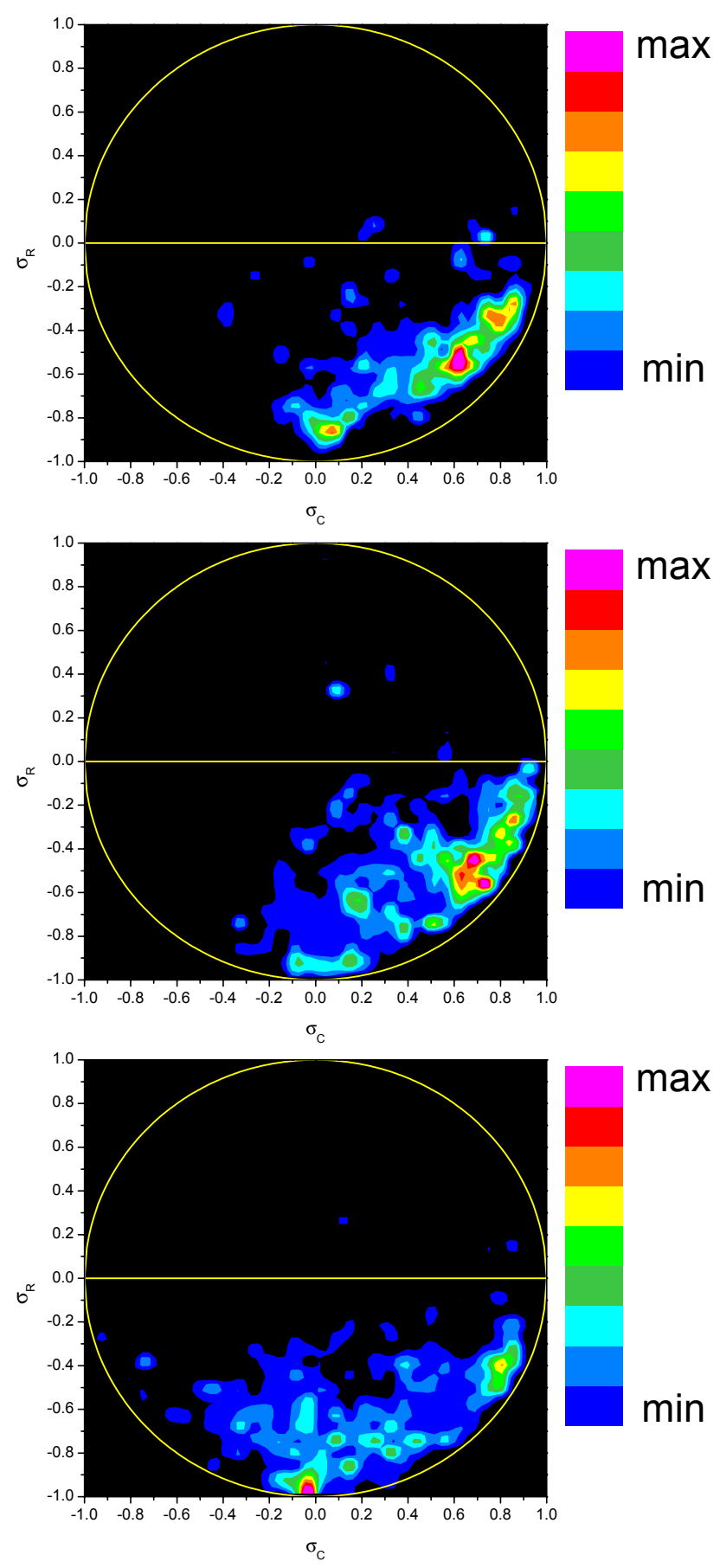

Fig. 8. From top to bottom: frequency histograms of $\sigma_{R}$ versus $\sigma_{C}$ for slow wind observed by Helios 2 at $0.32,0.69$ and $0.90 \mathrm{AU}$, respectively. The color code, for each panel, is normalized to the maximum of the distribution.

at $0.3 \mathrm{AU}$ are not characterized by a single dominant peak as it was the case for fast wind. Moreover, the radial evolution is much less impressive than that for the fast wind. At $0.3 \mathrm{AU}$ the main body of the distribution lies along the $\sigma_{C} \sim 0.5$ line and ends up at $\sigma_{C} \sim 0$ by the time the slow wind reaches $0.88 \mathrm{AU}$. Consequently, also the $\sigma_{R}-\sigma_{C}$ histograms in Fig. 8 do not show a striking radial evolution. Although high values of $\sigma_{C}$ can be encountered also within slow wind they are statistically much less relevant than in fast wind and a well defined population characterized by $\sigma_{C} \sim 0$ and $\sigma_{R} \sim-1$, already present at $0.3 \mathrm{AU}$, becomes one of the dominant peaks of the histogram when the wind reaches $0.88 \mathrm{AU}$. This last feature represents the most striking difference with what happens in fast wind. It is also worth noticing that, when the wind reaches $0.88 \mathrm{AU}$, some population with negative $\sigma_{C}$ clearly appears. However, the highly negative value of the associated $\sigma_{R}$ suggests that these elements cannot be considered as inward propagating Alfvén modes but rather as advected structures.

Figure 9, in the same format of Fig. 5, shows that highly negative values of $\sigma_{R}$ are generalized and not exclusively related to any particular combination of $e^{+}$and $e^{-}$, i.e. any particular value of $\sigma_{C}$, no matter what the heliocentric distance might be. On the other hand, values of $\sigma_{R}$ close to 0 sporadically appear at all distances. This result confirms that in slow wind magnetic energy always dominates the fluctuations (see references in Bruno and Carbone, 2005) but rules out the possibility that this is solely due to an increased presence of MFDTs (Tu and Marsch, 1993).

Finally, we like to underline that results, entirely similar to those shown so far in the present paper, have been obtained analyzing Helios 1 observations during its first solar mission in 1975 between 0.3 and $1 \mathrm{AU}$. However, we do not show them for sake of brevity.

\subsection{WIND observations at $1 \mathrm{AU}$}

To corroborate the results shown in the previous section with a more robust statistics, we employed WIND s/c data recorded for 14 months from the beginning of 1995 to day 121 of 1996 . The data used in this analysis are $240 \mathrm{~s}$ averages of solar wind magnetic field and plasma. We removed all the magnetospheric crossings which represented about $7 \%$ of the selected time interval. The available number of $240 \mathrm{~s}$ averages used for the analysis is then slightly more than 162000 .

Proceeding like we did for Helios data, we computed the same quantities described in Sect. 2.1. During the time interval within which WIND's data were analyzed, the solar wind was characterized mostly by corotating high velocity streams as expected for a typical minimum phase of the solar cycle (Bruno and Carbone, 2005). As suggested by Helios' results in Sect. 2.1, we performed two analyses for fast and slow wind, separately (see Table 1 for details). For the fast wind we set up a lower speed limit of $550 \mathrm{~km} / \mathrm{s}$ while, for the slow wind, we set up an upper speed limit of $400 \mathrm{~km} / \mathrm{s}$.

Results relative to fast wind are shown in Fig. 10 where, the top panel, shows the 2-D histogram for $\log e^{-}$and $\log e^{+}$in the same format of Fig. 1, the lower panel shows the relative $\sigma_{R}-\sigma_{C}$ histogram and the bottom panel shows the average value of $\sigma_{R}$ computed inside each square bin 

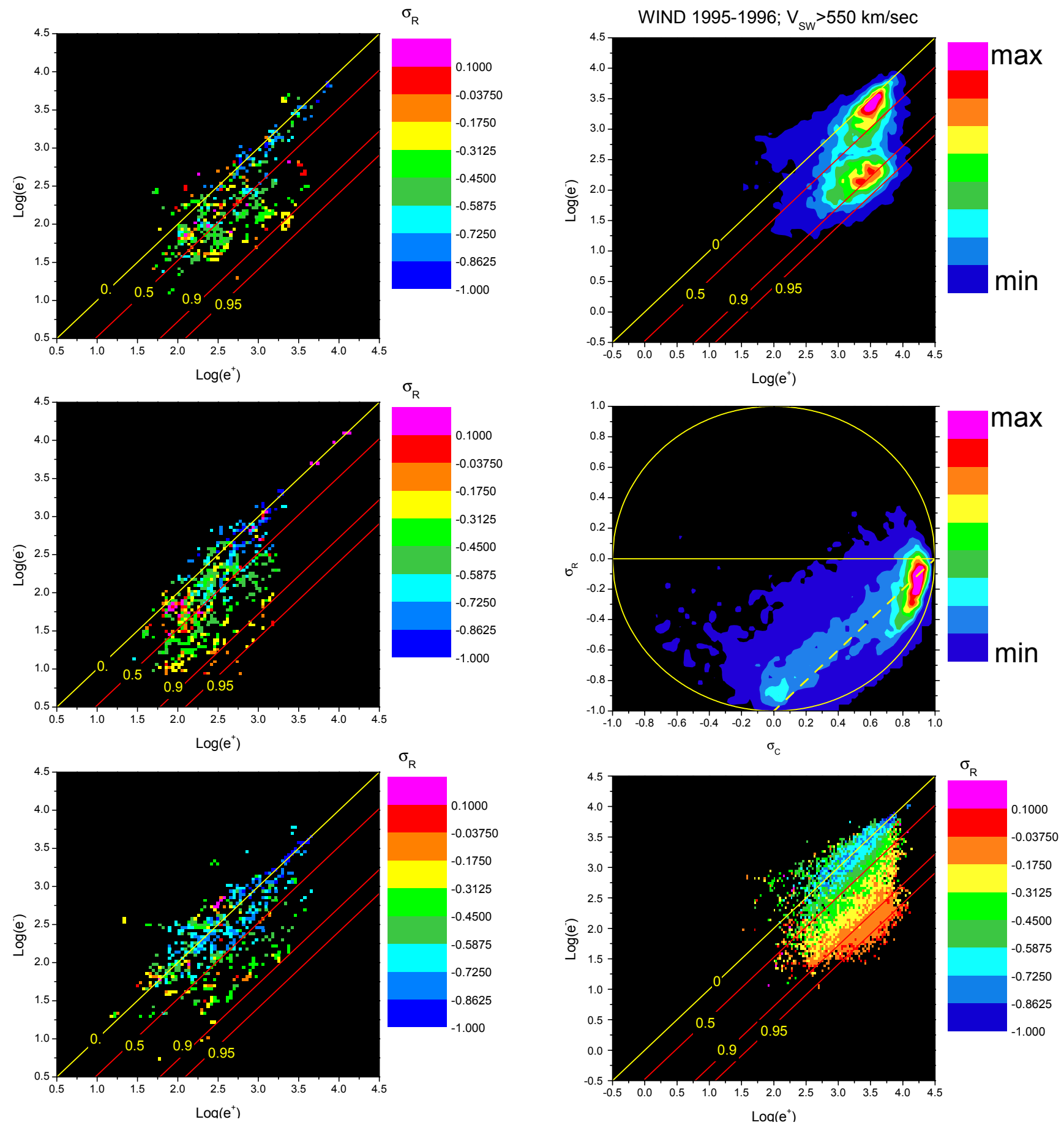

Fig. 9. From top to bottom: distribution of the average value of $\sigma_{R}$ computed within each square bin $\log \left(e^{-}\right) \times \log \left(e^{+}\right)$for slow wind observed by Helios 2 at $0.32,0.69$ and $0.90 \mathrm{AU}$, respectively. The color code, for each panel, indicates the interval value of $\sigma_{R}$.

$\log \left(e^{-}\right) \times \log \left(e^{+}\right)$in the same format of Fig. 5. Also in this case, as it already happened for the fast wind observed by $\mathrm{He}$ lios at $0.88 \mathrm{AU}$, fluctuations appear to be divided into 2 main populations, namely Alfvénic fluctuations and fluctuations

Fig. 10. Fast wind, with speed larger than $550 \mathrm{~km} / \mathrm{s}$, observed by WIND s/c during 1995 and the first 4 months of 1996. From top to bottom: frequency histograms of $\log \left(e^{-}\right)$versus $\log \left(e^{+}\right)$, frequency histograms of $\sigma_{R}$ versus $\sigma_{C}$, distribution of the average value of $\sigma_{R}$ computed within each square bin $\log \left(e^{-}\right) \times \log \left(e^{+}\right)$, respectively.

magnetically dominated. In order to evaluate the relevance of this second population we can adopt the same limits for 

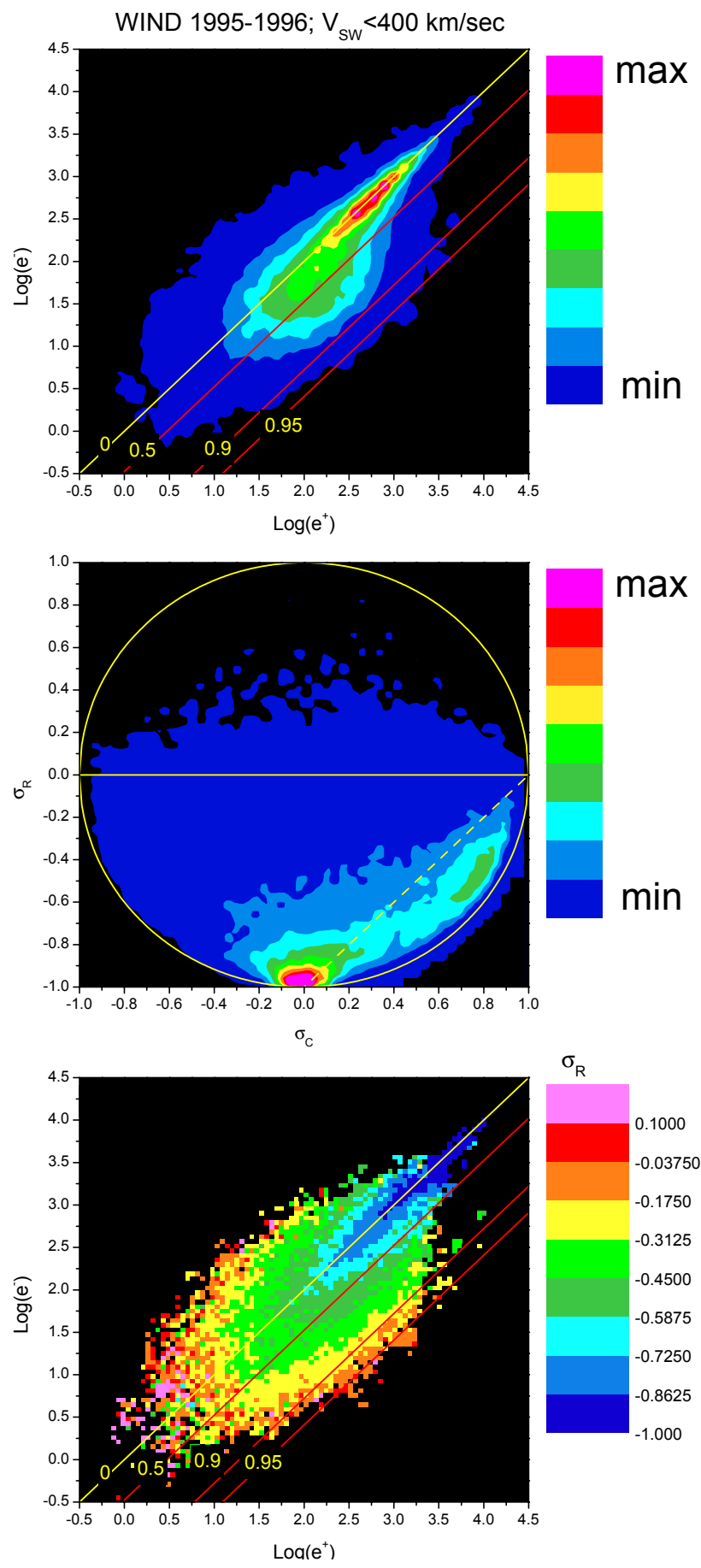

Fig. 11. Same format as of Fig. 10 but referring to slow wind, with speed less than $400 \mathrm{~km} / \mathrm{s}$.

$\sigma_{C}$ and $\sigma_{R}$ that we used for Helios observations at $0.88 \mathrm{AU}$ within fast wind and we end up with a rough estimate around $25 \%$ of the total number, not far from the rough estimate we made in Sect. 2.1. Finally, it is worth noticing that the distribution shown in the middle panel has a sort of ridge which is well fitted by the yellow dashed line referring to Eq. (2). This suggests that in some cases the simple model proposed by Tu and Marsch (1993) could be able to reproduce the observed situation to a good level and deserves some more careful and extensive analysis which cannot be done in this paper.

Next Fig. 11, in the same format of Fig. 10, shows the results obtained for the slow wind, i.e. solar wind speed lower than $400 \mathrm{~km} / \mathrm{s}$. The distribution in the top panel is dominated by an elongated peak centered around $\sigma_{C}=0$ but located at high values of $e^{-}$and $e^{+}$. Moreover, the same distribution shows a bulge extending towards higher values of $\sigma_{C}$. The lower panel clearly shows that the highest peak of the distribution belongs to a family characterized by $\sigma_{C} \simeq 0$ and $\sigma_{R} \simeq-1$. On the other hand, the Alfvénic population, which dominates the fast wind, has almost disappeared. However, the bottom panel clearly suggests that those events strongly dominated by magnetic energy are mainly due to the largest values of $e^{-}$and $e^{+}$as indicated by the color code. Also in this case, similarly to what we found for Helios 2 within fast wind (see Fig. 6) studying the compressive character of the fluctuations, WIND observations reveal that the lowest level of magnetic compression corresponds to those two populations that we identifies as Alfvénic fluctuations and magnetically dominated events (Fig. 12). In particular, the general level of compression appears larger than in the Helios 2 because our selection of WIND's time intervals was done automatically after we set up a threshold in the speed and, consequently, some intervals belonging to stream-stream interface or other compressive regions might have been included. However, also in this case, the lower panel indicates that most of the total vector variability is due to directional rather than compressive fluctuations.

\subsection{Ulysses observations out of the ecliptic}

A further confirmation of the existence of two dominating distinct families of MHD fluctuations in the solar wind comes from Ulysses observations at high latitude. As a matter of fact, these observations, together with those relative to the ecliptic, provide a complete picture in the 3-D heliosphere. The time period we chose for Ulysses spans from day 190 to day 330 of 1994. During this period of time the radial distance of the s/c was between 2.75 and $1.77 \mathrm{AU}$, the heliographic latitude varied between $-72^{\circ}$ and $-62^{\circ}$ with a maximum excursion at $-80^{\circ}$ and the $\mathrm{s} / \mathrm{c}$ was constantly immersed in a high velocity wind flowing at an average speed of $\sim 745 \mathrm{~km} / \mathrm{s}$ (see Table 1). The data set consists of $8 \mathrm{~min}$ averages of magnetic field and velocity measurements. This analysis follows the steps of the analysis made by Bavassano et al. (1998) but it is limited to fast polar wind observed at the highest negative latitudes, choosing an interval of time similar to that analyzed by Bavassano et al. (2005) who selected the three southernmost solar rotation seen by Ulysses in 1994.

Figure 13, relative to Ulysses observations, not only confirms previous results (Bavassano et al., 1998) but it also 


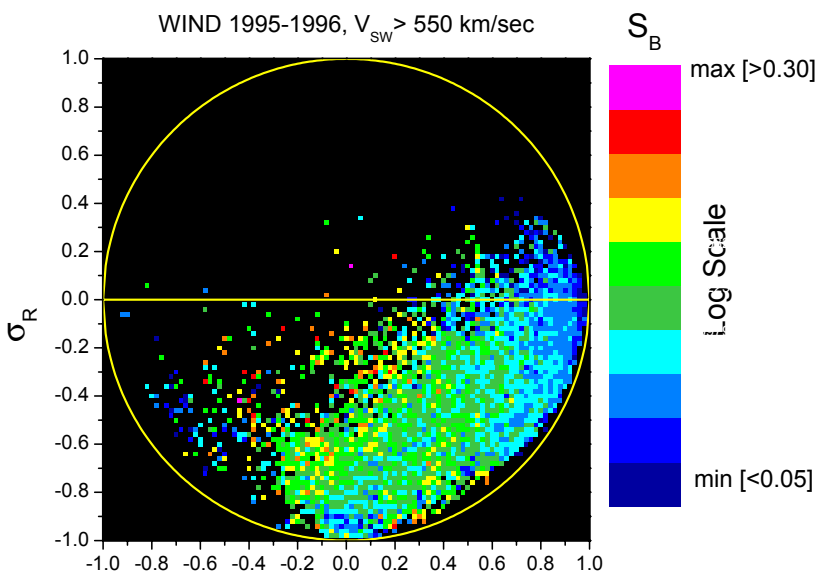

$\sigma_{\mathrm{C}}$

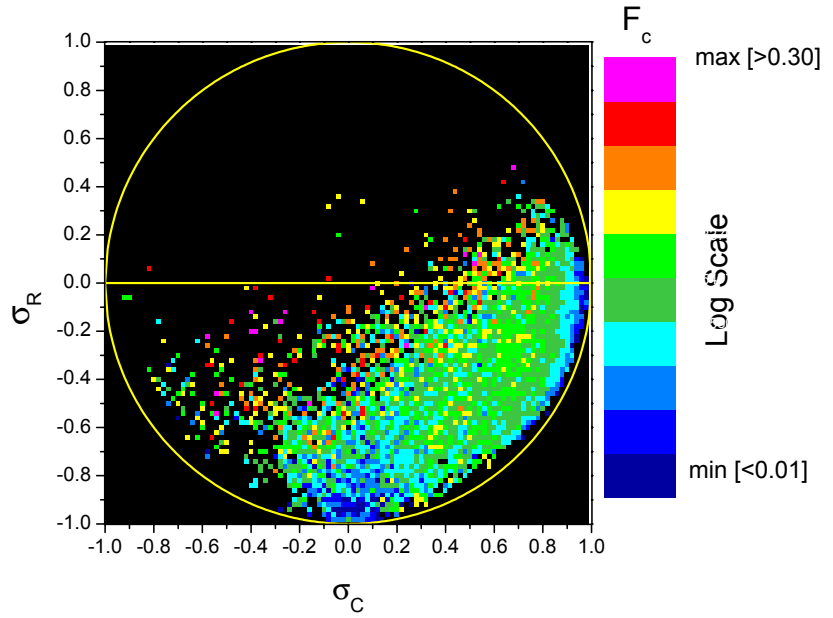

Fig. 12. From top to bottom: average value of $S_{B}$ and $F_{C}$ (see text for definitions) computed within each square bin $\sigma_{R} \times \sigma_{C}$ for WIND observations within fast wind, respectively.

strengthens the results we just obtained from Helios 2 and WIND observations. The top panel of this figure highlights again the presence of two main populations. One located close to $\sigma_{C}=0$ line and the other one at higher values of $\sigma_{C}$. The lower two panels help to disclose the nature of these two different families which are, again, magnetically dominated and Alfvénic fluctuations. These results resemble much more those obtained from Helios 2 at 0.88 AU and WIND at $1 \mathrm{AU}$ rather than those relative to Helios 2 at $0.3 \mathrm{AU}$ indicating that polar fast wind at roughly $2 \mathrm{AU}$ is more evolved than ecliptic fast wind around $0.3 \mathrm{AU}$, as abundantly discussed in literature (see review by Bruno and Carbone, 2005, and paper by Matthaeus et al., 1998). However, it should also be remarked that the Alfvénic peak of the distribution is characterized by a larger negative value of $\sigma_{R}$ with respect to Helios 2 at $0.88 \mathrm{AU}$ and WIND at $1 \mathrm{AU}$. At first sight, this feature might be attributed to the fact that, in this case, as generally done in literature (Bruno and Carbone, 2005), the pressure anisotropy factor $F$, contained in the conversion factor used
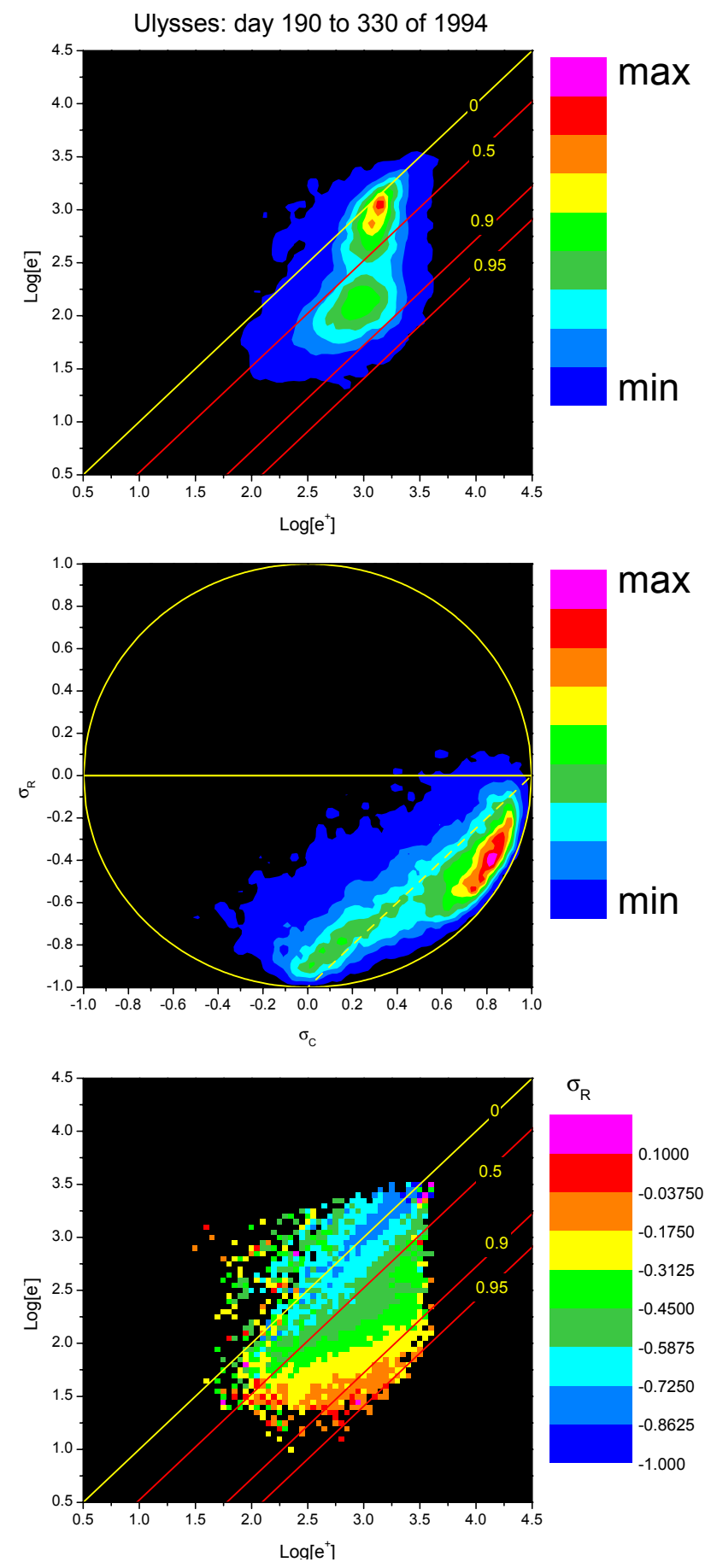

Fig. 13. Polar wind observed by Ulysses s/c during the south passage of 1994. From top to bottom: frequency histograms of $\log \left(e^{-}\right)$ versus $\log \left(e^{+}\right)$, frequency histograms of $\sigma_{R}$ versus $\sigma_{C}$, distribution of the average value of $\sigma_{R}$ computed within each square bin $\log \left(e^{-}\right) \times \log \left(e^{+}\right)$, respectively.

to transform magnetic into velocity fluctuations (Belcher and Davis Jr., 1971), i.e. $\boldsymbol{v}=( \pm F / \sqrt{4 \pi \rho}) \boldsymbol{b}$, is considered equal to 1 . However, this fact has already been noticed and largely 


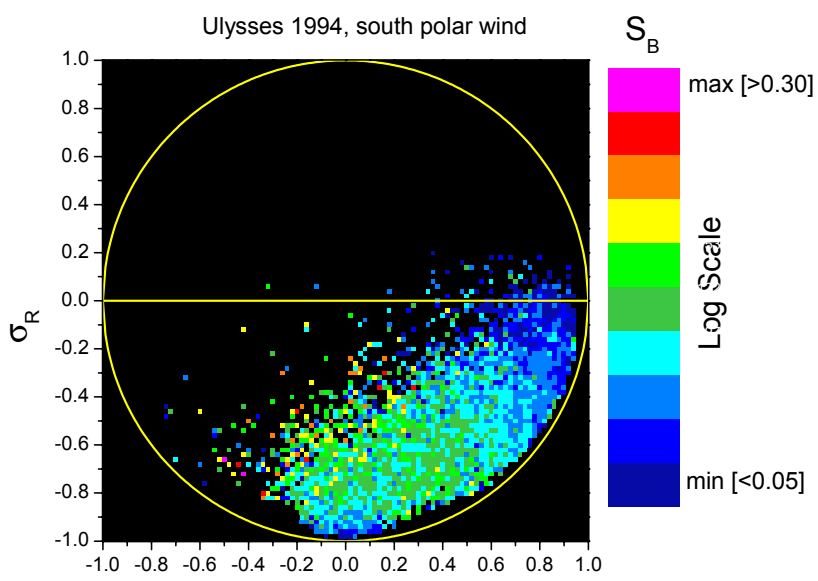

$\sigma_{\mathrm{C}}$

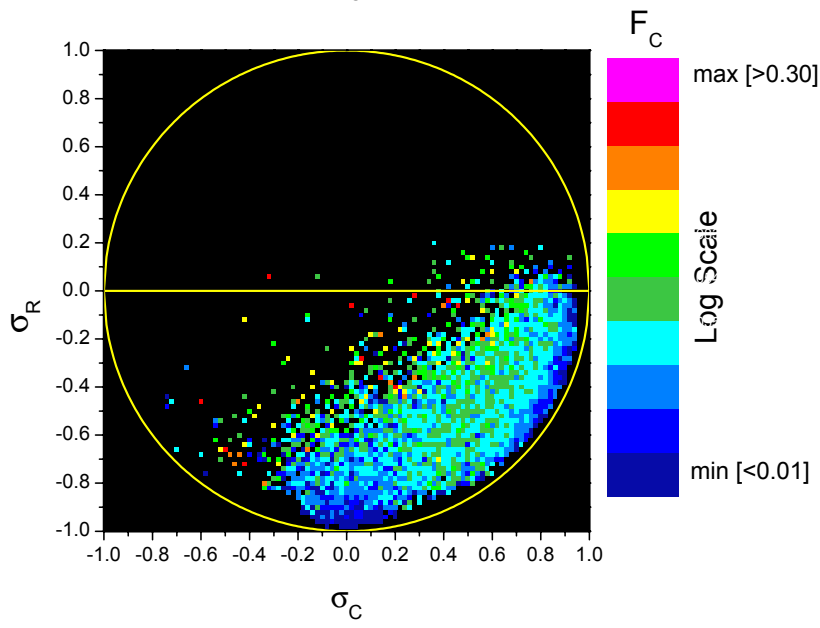

Fig. 14. From top to bottom: average value of $S_{B}$ and $F_{C}$ (see text for definitions) computed within each square bin $\sigma_{R} \times \sigma_{C}$ for Ulysses observations within the south polar wind, respectively. .

discussed by Bavassano and Bruno (2000) who accurately considered all effects due to total mass density, total thermal anisotropy, and ion differential streaming for a plasma made of protons, alpha particles and electrons. Their results clearly indicated that, in the inner heliosphere, the radial variation of the Alfvén ratio $r_{A}$ towards values less than 1 (i.e. $\sigma_{R}<0$ ) is not completely due to a missed inclusion of multi-fluid effects in the conversion from magnetic field to Alfvén units. On the other hand, Goldstein et al. (1995), discussing Ulysses results, suggested that the factor $F$ in the Alfvén relation might be sensitive to interstellar pick-up ions. As a matter of fact, these ions might appreciably influence the pressure anisotropy and, consequently, using $F=1$, as usually done in literature, would enhance the power associated to magnetic field fluctuations. Thus, this effect, disregardable in the inner heliosphere, might play a role in the case of Ulysses. Several other hypotheses have been formulated in literature to explain the radial behaviour of $r_{A}$ and we invite the reader to take a look at the references related to this topic and reported in the review by Bruno and Carbone (2005). Thus, if pick-up ions play a significant role in case of Ulysses, we might ascribe to this phenomenon the fact that the yellow dashed line, which represents the Tu and Marsch (1991) model, completely misses the Alfvénic population. However, we also believe that the linear superposition of Alfvénic fluctuations and MFDTs, represented by relation (2), is not generally encountered in the solar wind and is an oversimplification of the real situation.

At this point we can attempt an estimate of the relative number of magnetic structures with respect to the whole data set. Adopting the same procedure used in the previous Sects. 2.1 and 2.2, that means counting only those elements with $\sigma_{C}<0.5$ and $\sigma_{R}<-0.6$, we end up with a population of $27 \%$ of the total number, again, of the same order of the rough estimates we made for Helios 2 and WIND.

Finally, the two panels of Fig. 14, in the same format of Figs. 12 and 6, confirm that, also for Ulysses in the polar wind, the two main populations found by the present analysis are scarsely compressive as expected for Alfvénic fluctuations and MFDTs (Tu and Marsch, 1991, 1993).

One more step towards the characterization of these magnetic structures would be that of investigating how these events are distributed in time. If they were independent on each other we would expect to find a Poissonian distribution of the waiting times but, if some long range correlations existed we would find a power-law like distribution (Bruno and Carbone, 2005). Keeping the limits for $\sigma_{C}$ and $\sigma_{R}$ that we used to locate these events, we measured the time elapsed, i.e. the waiting time (WT, herefater), between the end of one event and the beginning of the next one. Obviously, the duration of each event is determined by the number of averages during which $\sigma_{C}<0.5$ and $\sigma_{R}<-0.6$. We performed this kind of analysis only for Ulysses data set because of more robust statistics with respect to Helios 2 and because of a better continuity in the data coverage with respect to WIND (see Sect. 2.2).

The distribution of the normalized frequency of the WTs, expressed in minutes, is shown in Fig. 15. This distribution is clearly neither a power nor an exponential as demonstrated by the blue solid line representing the best possible fit obtained using a Poissonian curve $y=(B / \lambda) e^{-\frac{t}{\lambda}}$.

On the contrary, a distribution like

$y=A x^{\alpha} e^{-\frac{t}{t_{c}}}$

which takes into account both contributions, i.e. the power law and the Poissonian, does a very good job as indicated by the solid red line. This fact suggests that the shortest magnetic structures keep a sort of memory of each other and that this long range correlation fades away as we look at events of this kind separated by longer and longer time intervals. Just for sake of completeness, we report the values obtained from the fitting procedure: $A=49.05 \pm 9.13, \alpha=-1.04 \pm 0.05, t_{c}=412.05 \pm 62.54$. 
In particular, the value of the cutoff time $t_{c}$ indicates that long term correlations loose strength for intervals longer than about $7 \mathrm{~h}$.

\section{Conclusions}

The present analysis showed that magnetically dominated structures represent a remarkable component of the interplanetary MHD fluctuations. In fact, these structures and Alfvénic fluctuations dominate at scales typical of MHD turbulence. A rough estimate would suggest that more than $20 \%$ of all analyzed intervals of $1 \mathrm{~h}$ scale are magnetically dominated and weakly Alfvénic. Observations in the ecliptic and out of the ecliptic showed that these advected, mostly uncompressive structures are ubiquitous in the heliosphere and can be found in both fast and slow wind. On the contrary, the same analysis confirmed that Alfvénic fluctuations outwardly propagating strongly characterize fast wind but are rather negligible in slow wind, as we already know from literature (see review by Bruno and Carbone, 2005). In particular, Helios 2 and Helios 1 (results from the latter s/c have not been shown in the present paper for sake of brevity) observations revealed a clear radial dependence of these magnetic structures within fast wind, but not within slow wind. At short heliocentric distances $(\sim 0.3 \mathrm{AU})$ the turbulent population is largely dominated by Alfvénic fluctuations characterized by high values of $\sigma_{C}$ and a rather good level of energy equipartition $\left(\sigma_{R} \sim 0\right)$. However, as the wind expands, a new-born population, characterized by lower values of $\sigma_{C}$ and higher negative values of $\sigma_{R}$ becomes visible. Helios observations show that a new peak, located at $\sigma_{C} \sim 0$ and $\sigma_{R} \sim-1$, clearly appears in the distributionby the time the s/c reaches $1 \mathrm{AU}$.

As a matter of fact, results from the analysis of Ulysses' fast polar wind and WIND's fast wind well resemble and corroborate Helios results obtained in proximity of $1 \mathrm{AU}$. Moreover, we showed that, within fast wind, these magnetic structures well mimic $e^{-}$modes as it was stressed by $\mathrm{Tu}$ and Marsch $(1991,1993)$ in the case of MFDTs. As a matter of fact, most of the magnetic structures that we find share similar features with the MFDTs described by these authors although we cannot exclude that contributions to lower values of $\sigma_{R}$ might also come from simple Tangential Discontinuities which are ubiquitous in the solar wind.

Now, the question is whether or not these structures are created during the turbulent evolution of the fluctuations or they are already there at $0.3 \mathrm{AU}$ but too small compared to Alfvénic fluctuations to be seen. The fact that they are always present within slow wind already at $0.3 \mathrm{AU}$, where Alfvénic fluctuations are rather small, make us favor the solar origin of these structures. The reason why we do not see them at $0.3 \mathrm{AU}$ is due to the fact that these fluctuations, mainly non-compressive, change the direction of the magnetic field similarly to Alfvénic fluctuations but producing a much smaller effect. As the wind expands, the Alfvénic
Ulysses waiting time statistics for MFDTs

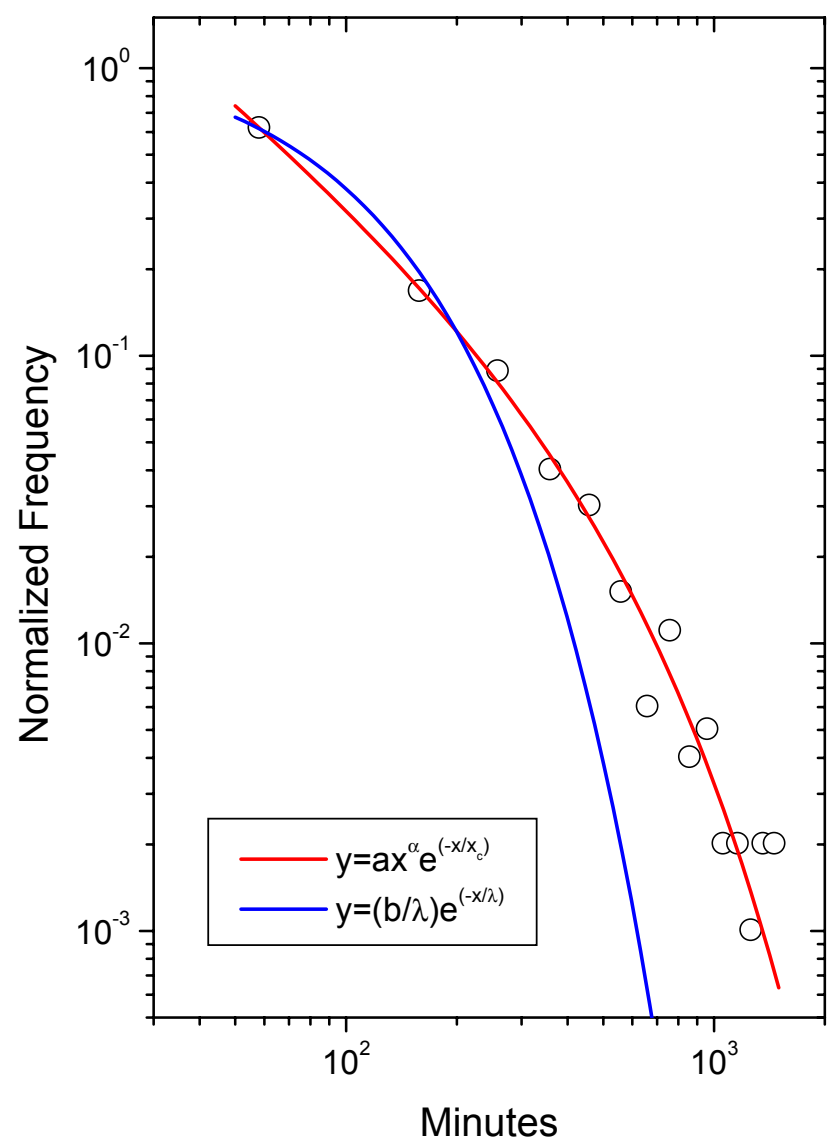

Fig. 15. Waiting time statistics for MFDTs events (see text for selection criteria) observed by Ulysses. Frequency has been normalized to the total number of events. The blue and red curves are the best fits to the data, obtained using the relative expressions listed in the inset.

component undergoes non-linear interactions which produce a transfer of energy to smaller and smaller scales while, these structures, being advected, have a much longer lifetime. As the expansion goes on, the relative weight of these fluctuations grows and they start to be detected in our analysis. To this regard, Tu and Marsch (1992) and Tu and Marsch (1993) suggested that large scale variations along the magnetic field lines might be intermingled with small scale variations perpendicular to these lines, being the former static structures advected by the wind and the latter Alfvénic fluctuations. Depending on the relative direction in which the observer samples the solar wind parameters with respect to the background magnetic field a different mixture of Alfvénic fluctuations and static structures will contribute to the resulting value of $\sigma_{C}$ and $\sigma_{R}$. This angular effect would be more and more important with increasing heliocentric distance because of the spiral configuration of the interplanetary 
magnetic field and of the larger radial damping of Alfvénic fluctuations compared to advected structures. These structures would represent a specific kind of the 2-D turbulence (Tu and Marsch, 1993) reported by Matthaeus et al. (1990). Following (Tu and Marsch, 1993), MFDTs would arise naturally like the Alfvénic fluctuations since they obey the MHD equations because of the following properties: $\boldsymbol{B} \cdot \nabla \boldsymbol{B}=0$ and $\boldsymbol{V},|\boldsymbol{B}|, \rho$ all constant (Tu and Marsch, 1991). While at short heliocentric distances 2-D turbulence might be of the standard type since $\boldsymbol{B}_{0} \gg \delta \boldsymbol{b}$, MFDTs could be the final state of a radial fast evolution experienced by these fluctuations within the first layers of the solar corona (Tu and Marsch, 1993).

Interesting enough is the the theoretical model presented by Chang et al. (2004) which tells us that propagating modes and coherent, advected structures are both necessary, inseparable ingredients of MHD turbulence, since they share a common origin within the general view described by the physics of complexity (Chang, 2003; Chang et al., 2004). Propagating modes experience resonances which generate coherent structures which, in turn, will migrate, interact and eventually generate new modes.

Our interpretation is that at least part of these magnetic structures might also be a specific, uncompressive, signature of the crossing of the border between adjacent flux tubes forming, as suggested by Bruno et al. (2001); Bruno et al. (2004); Bruno and Carbone (2005), the advected background structure of the interplanetary magnetic field, strongly related to the complex magnetic topology at the sun's surface. However, a more specific study is needed to prove our interpretation since these structures may not necessarily occur near the border of a stream tube, as remarked by Tu and Marsch (1991). Finally, we like to recall that the waiting time statistics elaborated for Ulysses observations showed that these structures might hide some long-range correlation, i.e. a sort of "memory", which would make the underlying generating process non-Poissonian, possibly a cascading process.

Whatever the nature of these fluctuations might be, we showed that they represent a remarkable fraction of all fluctuations. Our statistical study provides a rough estimate of the importance of magnetically dominated structures in the solar wind turbulence.

Acknowledgements. Helios 2 magnetic field $81 \mathrm{~s}$ averages derive from the Rome-GSFC magnetic experiment (P. I. S. F. Mariani and N. F. Ness). Plasma data form the same s/c derive from the solar wind analyzer and were provided by H. Rosenbauer and R. Schwenn, PIs of the experiment. The use of data of the solar wind plasma analyzer (P. I. D. J. McComas, Southwest Research Institute, San Antonio, Texas, USA) and of the magnetometers (P. I. A. Balogh) aboard the Ulysses spacecraft is gratefully acknowledged. We also acknowledge the use of data of the solar wind plasma analyzer (P. I. K. Ogilvie, NASA-GSFC, MD, USA) and of the magnetometers (P. I. R. Lepping) aboard the WIND spacecraft The data have been made available by the World Data Center A for Rockets and Satellites (NASA/GSFC, Greenbelt, Maryland, USA). We also thank E. Pietropaolo for computer assistance. The present work has been supported by the Italian Space Agency (ASI) under contract \#035/05/0.

Topical Editor B. Forsyth thanks M. Goldstein and S. Oughton for their help in evaluating this paper.

\section{References}

Bavassano, B. and Bruno, R.: Velocity and magnetic field fluctuations in Alfvnic regions of the inner solar wind: Three-fluid observations, J. Geophys. Res., 105, 5113-5118, 2000.

Bavassano, B., Dobrowolny, M., Fanfoni, G., Mariani, F., and Ness, N.: Statistical properties of MHD fluctuations associated with high-speed streams from Helios 2 observations, Solar. Phys., 78, 373-384, 1982a.

Bavassano, B., Dobrowolny, M., Mariani, F., and Ness, N.: Radial evolution of power spectra of interplanetary Alfvénic turbulence, J. Geophys. Res., 87, 3617-3622, $1982 b$.

Bavassano, B., Pietropaolo, E., and Bruno, R.: Cross-helicity and residual energy in solar wind turbulence. Radial evolution and latitudinal dependence in the region from 1 to $5 \mathrm{AU}, \mathrm{J}$. Geophys. Res., 103, 6521-6530, 1998.

Bavassano, B., Pietropaolo, E., and Bruno, R.: Alfvénic turbulence in the polar wind: A statistical study on cross helicity and residual energy variations, J. Geophys. Res., 105, 12 697-12 704, 2000.

Bavassano, B., Bruno, R., and D'Amicis, R.: Large-scale velocity fluctuations in polar solar wind, Ann. Geophys., 23, 1025-1031, 2005 , http://www.ann-geophys.net/23/1025/2005/.

Belcher, J. and Davis Jr., L.: Large-amplitude Alfvén waves in the interplanetary medium, J. Geophys. Res., 76, 3534-3563, 1971.

Bieber, J., Wanner, W., and Matthaeus, W.: Dominant twodimensional solar wind turbulence with implications for cosmic ray transport, J. Geophys. Res., 101, 2511-2522, 1996.

Bruno, R. and Bavassano, B.: Origin of low cross-helicity regions in the solar wind, J. Geophys. Res., 96, 7841-7851, 1991.

Bruno, R. and Bavassano, B.: Cross-helicity depletions in the inner heliosphere, and magnetic field and velocity fluctuation decoupling, Planet. Space Sci., 41, 677-685, 1993.

Bruno, R. and Carbone, V.: The Solar Wind as a Turbulence Laboratory, Living Reviews in Solar Physics, 2, 2005.

Bruno, R., Carbone, V., Veltri, P., Pietropaolo, E., and Bavassano, B.: Identifying intermittent events in the solar wind, Planetary Space Sci., 49, 1201-1210, 2001.

Bruno, R., Carbone, V., Primavera, L., Malara, F., Sorriso-Valvo, L., Bavassano, B., and Veltri, P.: On the probability distribution function of small-scale interplanetary magnetic field fluctuations, J. Geophys. Res., 22, 3751-3769, 2004.

Chang, T.: Complexity Induced Plasma Turbulence in Coronal Holes and the Solar Wind, in: AIP Conf. Proc. 679: Solar Wind Ten, edited by: Velli, M., Bruno, R., and Malara, F., pp. 481-484, 2003.

Chang, T., Tam, S., and Wu, C.: Complexity induced anisotropic bimodal intermittent turbulence in space plasmas, Phys. Plasmas, 11, 1287-1299, 2004.

Dobrowolny, M., Mangeney, A., and Veltri, P.: Properties of magnetohydrodynamic turbulence in the solar wind, Astron. Astrophys., 83, 26-32, 1980. 
Elsässer, W.: The hydromagnetic equations, Phys. Rev., 79, 183, 1950.

Goldstein, B. E., Neugebauer, M., and Smith, E. J.: Alfvén waves, alpha particles, and pickup ions in the solar wind, Geophys. Res. Lett., 22, 3389-3392, doi:10.1029/95GL03182, 1995.

Goldstein, M., Roberts, D., and Matthaeus, W.: Systematic errors in determining the propagation direction of interplanetary Alfvénic fluctuations, J. Geophys. Res., 91, 13 357-13 365, 1986.

Grappin, R., Mangeney, A., and Marsch, E.: On the origin of solar wind turbulence:Helios data revisited, in: Proceedings of the workshop on Turbulence and Non-linear Dynamics in MHD flows, edited by: Meneguzzi, M., Pouquet, A., and Sulem, P., pp. 81-86, North-Holland, 1989.

Horbury, T. S., Forman, M. A., and Oughton, S.: Spacecraft observations of solar wind turbulence: an overview, Plasma Physics and Controlled Fusion, 47, B703-B717, doi:10.1088/ 0741-3335/47/12B/S52, 2005.

Klein, L., Bruno, R., Bavassano, B., and Rosenbauer, H.: Anisotropy and minimum variance of magnetohydrodynamic fluctuations in the inner heliosphere, J. Geophys. Res., 98, 17461-17 466, 1993.

Marsch, E. and Tu, C.-Y.: Dynamics of correlation functions with Elsässer variables for inhomogeneous MHD turbulence, J. Plasma Phys., 41, 479-491, 1989.

Matthaeus, W., Goldstein, M., and Roberts, D.: Evidence for the presence of quasi-two-dimensional nearly incompressible fluctuations in the solar wind, J. Geophys. Res., 95, 20 673-20683, 1990.

Matthaeus, W. H., Smith, C. W., and Oughton, S.: Dynamical age of solar wind turbulence in the outer heliosphere, J. Geophys. Res., 103, 6495-6502, 1998.

Montgomery, D.: Major disruptions, inverse cascades, and the Strauss equations, Phys. Scr., 2, 83-88, 1982.

Müller, W.-C. and Grappin, R.: Spectral Energy Dynamics in Magnetohydrodynamic Turbulence, Phys. Rev. Lett., 95, $114502-$ $114505,2005$.

Oughton, S., Priest, E. R., and Matthaeus, W. H.: The influence of a mean magnetic field on three-dimensional magnetohydrodynamic turbulence, J. Fluid Mech., 280, 95-117, 1994.

Roberts, D.: Observation and simulation of the radial evolution and stream structure of solar wind turbulence, in: Solar Wind Seven Colloquium, pp. 533-538, 1992.

Roberts, D., Goldstein, M., Klein, L., and Matthaeus, W.: The nature and evolution of magnetohydrodynamic fluctuations in the solar wind: Voyager observations, J. Geophys. Res., 92, 11 02111 040, 1987a.

Roberts, D., Goldstein, M., Klein, L., and Matthaeus, W.: Origin and evolution of fluctuations in the solar wind: HELIOS observations and Helios-Voyager comparisons, J. Geophys. Res., 92, 12 023-12 035, 1987b.
Roberts, D., Ghosh, S., Goldstein, M., and Matthaeus, W.: Magnetohydrodynamic simulation of the radial evolution and stream structure of solar-wind turbulence, Phys. Rev. Lett., 67, 37413744, 1991.

Roberts, D., Goldstein, M., Matthaeus, W., and Ghosh, S.: Velocity shear generation of solar wind turbulence, J. Geophys. Res., 97, 17 115-17 130, 1992.

Shebalin, J., Matthaeus, W., and Montgomery, D.: Anisotropy in MHD turbulence due to a mean magnetic field, J. Plasma Phys., 29, 525-547, 1983.

Smith, C.: The Geometry of Turbulent Magnetic Fluctuations at High Heliograpahic Latitudes, in: Solar Wind Ten, edited by: Velli, M., Bruno, R., and Malara, F., vol. 679 of AIP Conf. Proc., pp. 413-416, 2003.

Tsurutani, B. and Smith, E.: Interplanetary discontinuities: temporal variations and the radial gradient from 1 to $8: 5 \mathrm{AU}, \mathrm{J}$. Geophys. Res., 84, 2773-2787, 1979.

Tu, C., Roberts, D., and Goldstein, M.: Spectral evolution and cascade constant of solar wind Alfvénic turbulence, J. Geophys. Res., 94, 13 575-13 578, 1989.

Tu, C.-Y. and Marsch, E.: Transfer equations for spectral densities of inhomogeneous MHD turbulence, J. Plasma Phys., 44, 103122, 1990.

Tu, C.-Y. and Marsch, E.: A case study of very low cross-helicity fluctuations in the solar wind, Ann. Geophys., 9, 319-332, 1991, http://www.ann-geophys.net/9/319/1991/.

Tu, C. Y. and Marsch, E.: The evolution of MHD turbulence in the solar wind, in: Solar Wind Seven Colloquium, pp. 549-554, 1992.

Tu, C.-Y. and Marsch, E.: A model of solar wind fluctuations with two components: Alfvén waves and convective structures, J. Geophys. Res., 98, 1257-1276, 1993.

Tu, C.-Y. and Marsch, E.: MHD structures, waves and turbulence in the solar wind: Observations and theories, Space Sci. Rev., 73, $1-2,1995$.

Wanner, W. and Wibberenz, G.: A study of the propagation of solar energetic protons in the inner heliosphere, J. Geophys. Res., 98, 3513-3528, 1993.

Zank, G. and Matthaeus, W.: Waves and turbulence in the solar wind, J. Geophys. Res., 97, 17 189-17 194, 1992.

Zhou, Y. and Matthaeus, W.: Non-WKB evolution of solar wind fluctuations: A turbulence modeling approach, Geophys. Res. Lett., 16, 755-758, 1989. 